\title{
Efficient tumor transduction and antitumor efficacy in experimental human osteosarcoma using retroviral replicating vectors
}

\author{
Shuji Kubo ${ }^{1} \cdot$ Misato Takagi-Kimura ${ }^{1} \cdot$ Noriyuki Kasahara $\mathbb{D}^{2}$
}

Received: 1 April 2018 / Revised: 12 May 2018 / Accepted: 24 May 2018 / Published online: 25 July 2018

(c) The Author(s) 2018. This article is published with open access

\begin{abstract}
Retroviral replicating vectors (RRVs) have achieved efficient tumor transduction and enhanced therapeutic benefit in a wide variety of cancer models. Here, we evaluated two different RRVs derived from amphotropic murine leukemia virus (AMLV) and gibbon ape leukemia virus (GALV), which utilize different cellular receptors (PiT-2 and PiT-1, respectively) for viral entry, in human osteosarcoma cells. Quantitative RT-PCR showed that low levels of expression of both receptors were observed in normal and non-malignant cells. However, high PiT-2 (for AMLV) and low PiT-1 (for GALV) expression was observed in most osteosarcoma cell lines. Accordingly, AMLV expressing the green fluorescent protein gene infected and replicated more efficiently than GALV in most osteosarcoma cell lines. Furthermore, RRVs expressing the cytosine deaminase prodrug activator gene showed differential cytotoxicity that correlated with the results of viral spread. AMLVRRV-mediated prodrug activator gene therapy achieved significant inhibition of subcutaneous MG-63 tumor growth over GALV in nude mice. These data indicate that AMLV vectors predominate over GALV in human osteosarcoma cells. Moreover, our findings support the potential utility of the two RRVs in personalized cancer virotherapy on the basis of receptor expression.
\end{abstract}

\section{Introduction}

Osteosarcoma is the most common primary bone tumor, primarily affecting young children and adolescents [1-5]. It is an aggressive malignant tumor that typically metastasizes to the lungs at an early phase [3-5]. Despite reported increases in survival rates because of improvements in chemotherapy, patients with pulmonary metastases at the time of diagnosis have only a $20 \%$ survival rate $[2,3,5]$. Therefore, novel therapeutic paradigms are urgently required.

The use of oncolytic viruses capable of tumor-selective replication has shown considerable promise as a novel treatment strategy $[6,7]$. This is based on the observation that tumor cells have impaired antiviral responses making

Shuji Kubo

s-kubo@hyo-med.ac.jp

1 Unit of Molecular and Genetic Therapeutics, Institute for Advanced Medical Sciences, Hyogo College of Medicine, Nishinomiya, Japan

2 Departments of Cell Biology and Pathology, Sylvester Comprehensive Cancer Center, University of Miami, Miami, FL, USA them more sensitive to replicating viruses [8]. However, experimental gene therapy for osteosarcoma has utilized only a few oncolytic viruses in the development of replicating viruses, including adenovirus [9-13], Semliki Forest virus [14], and Maraba virus [15].

Recently, we demonstrated that retroviral replicating vectors (RRVs) show tumor selectivity because of their inherent and stringent specificity for mitotically active cells. In contrast to other viruses used in cancer virotherapy, RRVs are non-cytolytic, but can be engineered to carry prodrug activator genes, which mediate synchronized cell killing of infected tumor cells upon prodrug administration. Using RRVs expressing the yeast cytosine deaminase (CD) prodrug activator gene, we showed highly efficient killing of a wide variety of cancer cells both in vitro and in vivo upon administration of its prodrug, 5-fluorocytosine (5FC) [16-22]. Based on these promising preclinical results, a Phase 1 clinical trial for RRV vector-mediated prodrug activator gene therapy in patients with recurrent high grade glioma was recently conducted in the US and Europe, resulting in complete remission and potential survival benefits [23]. RRV are also currently being evaluated in an international Phase III trial for recurrent high grade glioma (NCT02414165). 
To date, we have developed two different RRVs derived from amphotropic murine leukemia virus (AMLV) and gibbon ape leukemia virus (GALV) [22, 24]. Although both AMLV and GALV belong to the gammaretroviruses, they have divergent host ranges and are not in the same interference class [25-27] because they employ different receptors to infect target cells [25, 27]. AMLV uses cellular receptor PiT2 (SLC20A2) while GALV uses PiT1 (SLC20A1); both are mammalian type III inorganic phosphate transporters present in all phyla, which function as ubiquitously expressed facilitators of phosphate uptake [25-27].

In the present study, we compared AMLV-derived and GALV-derived RRVs for prodrug activator gene therapy of experimental human osteosarcoma. Our results indicate the potential utility of AMLV-mediated prodrug activator gene therapy in the treatment of osteosarcoma, including when the tumor is not permissive for GALV.

\section{Materials and methods}

\section{PiT-1 and PiT-2 mRNA expression profile analysis}

Total RNA was extracted from semiconfluent cell cultures grown on $10 \mathrm{~cm}$ dishes using the Sepasol-RNA I Super G RNA extraction solution (Nacalai Tesque, Inc., Kyoto, Japan). It was then treated with DNase to remove genomic DNA contamination. Quantitative (q)PCR was used to examine the expression of PiT-1, PiT-2, and glyceraldehyde-3-phosphate dehydrogenase (GAPDH) mRNA with the TaqMan One-Step RT-PCR Master Mix Reagents kit (Applied Biosystems Japan Ltd., Tokyo, Japan) according to the manufacturer's instructions. Primers and TaqMan probes for PiT-1 (Hs00965587_m1), PiT-2 (Hs00198840_m1), and GAPDH (Hs99999905_m1) were purchased from Applied Biosystems Japan. Briefly, $20 \mathrm{ng}$ of total RNA was added to the reaction mixture containing $18 \mathrm{pmol}$ of each of the primers and $5 \mathrm{pmol}$ of probe, and amplified for one cycle of $48^{\circ} \mathrm{C}$ for $30 \mathrm{~min}$ and $95^{\circ} \mathrm{C}$ for $10 \mathrm{~min}$, followed by 50 cycles of $95^{\circ} \mathrm{C}$ for $15 \mathrm{~s}$ and $60{ }^{\circ} \mathrm{C}$ for $1 \mathrm{~min}$.

\section{Cell lines}

Normal human osteoblast cells (NHOsts) and their specific media were purchased from Lonza Japan, Inc. (Tokyo, Japan). Human dermal fibroblasts were purchased from Cell Systems Corporation (Kirkland, WA, USA) and were grown in Roswell Park Memorial Institute 1640 medium (Nacalai Tesque) supplemented with $10 \%$ fetal calf serum (FCS; HyClone, Logan, UT, USA). The human embryonic kidney 293 cell line (transformed by the $E 1$ gene of adenovirus type 5) (HEK293; Microbix, Toronto, Canada) [28] was cultured in Dulbecco's modified Eagle's medium (DMEM; Nacalai Tesque) supplemented with 10\% FCS. Human osteosarcoma cell lines HOS, MG-63, and Saos-2, were purchased from the RIKEN BioResearch Center (Tsukuba, Ibaraki, Japan), and MNNG-HOS and U2OS human osteosarcoma cell lines were obtained from the American Type Culture Collection (Manassas, VA, USA). These cells were grown in DMEM supplemented with $10 \%$ FCS. All cells were incubated at $37^{\circ} \mathrm{C} / 5 \% \mathrm{CO}_{2}$.

\section{Viral vector plasmid and virus production}

The RRV vector plasmids pAMLV-GFP, pGALV-GFP, pAMLV-CD, and pGALV-CD have been described previously [22]; each contains a full-length replicationcompetent amphotropic AMLV or GALV provirus with an additional internal ribosome entry site (IRES)-GFP or IRES-CD cassette, respectively (Figs. 2a and 3a). For virus production, 293T cells were transiently transfected with the vector plasmid using Lipofectamin 2000 (Life Technologies Japan, Tokyo, Japan), replenished with serum-free medium, then the supernatant was harvested $48 \mathrm{~h}$ later, filtered, and stored at $-80{ }^{\circ} \mathrm{C}[19,22]$. Vector titers were determined by fluorescent protein expression using a FACScalibur flow cytometer (Becton Dickinson Japan, Tokyo, Japan) and expressed as transducing units per $\mathrm{mL}$.

\section{In vitro replication kinetics of RRVs}

To analyze replication kinetics in vitro, virus vector stocks (AMLV-GFP and GALV-GFP) at a multiplicity of infection (MOI) of 0.01 were used to infect various human cell lines at $20 \%$ confluency. At serial time points, the cells were trypsinized, one-fourth of the cells were replated, and the remainder were analyzed for GFP expression by flow cytometry as described above.

\section{In vitro cytotoxicity assay}

To quantitatively analyze drug cytotoxicity, triplicate wells containing human MNNG-HOS, HOS, or MG-63 cells $\left(1 \times 10^{4}\right.$ cells/well) pretransduced with AMLV-CD or GALV-CD at an MOI of 0.01 and maintained for 15 days, were cultured in 96-well tissue culture plates with various concentrations of 5FC. On Day 3, viable cell numbers of triplicate cultures were measured by the AlamarBlue method according to the manufacturer's instructions (Alamar Biosciences, Inc., Sacramento, CA). Briefly, $40 \mu \mathrm{L}$ of AlamarBlue was aseptically added to the cultures, which were then returned to the incubator for $3 \mathrm{~h}$; fluorescence was measured by an ARVO X4 multilabel plate reader 
with a $544 \mathrm{~nm}$ excitation wavelength and a $590 \mathrm{~nm}$ emission wavelength (PerkinElmer Japan, Tokyo, Japan). The percentage of viable cells was determined by calculating the fluorescence of viable cells as measured against wells containing no $5 \mathrm{FC}$.

\section{Prodrug activator gene therapy in subcutaneous tumor models}

BALB/c-nu/nu (nude) mice (Charles River Japan Co., Yokohama, Japan) were bred and maintained under specific pathogen-free conditions. All studies followed protocols approved by the Hyogo College of Medicine Animal Research Committee. Human osteosarcoma xenografts were established in 5-week-old to 6-week-old female nude mice by subcutaneous inoculation of $1 \times 10^{6}$ MG-63 cells into the right dorsal flank. When tumors reached a diameter of $\sim 5-6 \mathrm{~mm}$, three groups of mice ( $n=10$ per group) were then injected intratumorally with $50 \mu \mathrm{L}$ of PBS, AMLV$\mathrm{CD}$, or GALV-CD on Day 0, followed by the intraperitoneal administration of $5 \mathrm{FC}(500 \mathrm{mg} / \mathrm{kg} /$ day $)$ three times a week from Day 14 to Day 34. The mice were observed closely and the tumors were measured twice a week. The tumor volume was calculated as $a \times b^{2} \times 0.5$, where $a$ and $b$ were the largest and smallest diameters, respectively.

\section{Statistical analysis}

The results are presented as mean $\pm \mathrm{SD}$. Statistical significances of differences were calculated using the Student's $t$-test, and a $P$-value of $<0.01$ was considered significant in all analyses.

\section{Results}

\section{mRNA expression of cellular receptors for RRVs}

We first analyzed the expression levels of cellular receptors for AMLV (PiT-2) and GALV (PiT-1) in human osteosarcoma cell lines by qPCR. In normal human primary NHOsts and fibroblasts and non-malignant human HEK293 cells, mRNA levels of both PiT-1 and PiT-2 (Fig. 1a, b) were relatively low, at less than 10.3-fold compared with fibroblasts. In most osteosarcoma cells (HOS, MG-63, Saos-2, and U2OS), low PiT-1 but high PiT-2 expression was observed. However, in MNNG-HOS cells, mRNA levels of both PiT-1 and PiT-2 were high (>10,000-fold compared with fibroblasts). These findings of low PiT-1 expression in non-malignant cells but high PiT-1 expression in most osteosarcoma cells suggested an advantage of AMLV over GALV for targeting human osteosarcoma to ensure safety and efficacy.

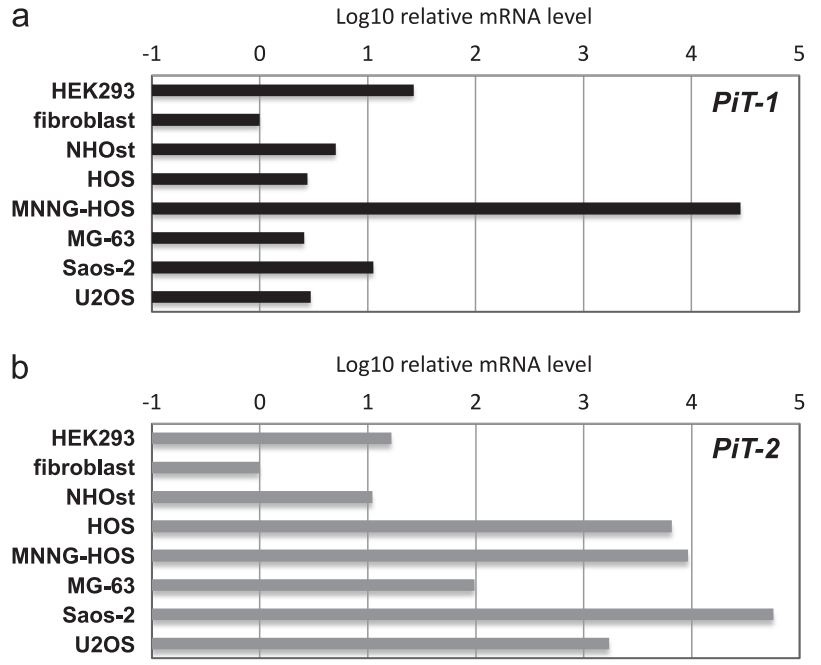

Fig. 1 Relative mRNA levels of PiT-1 and PiT-2 detected by quantitative RT-PCR. Total RNA was extracted from various human cells, including non-malignant human cells (HEK293 cells, fibroblasts, and NHOsts) and osteosarcoma cell lines (HOS, MNNG-HOS, MG-63, Saos-2, and U2OS). RNA was reverse-transcribed and amplified by PCR with specific primers for PiT-1 (a), PiT-2 (b), and GAPDH as a control for normalization

\section{AMLV vectors replicate efficiently in cultured human osteosarcoma cell lines}

We next compared the replication kinetics of AMLV-GFP and GALV-GFP in human osteosarcoma cells (Fig. 2a). Both RRVs efficiently spread up to $90 \%$ by 12 days after virus inoculation in non-malignant transformed HEK293 cells (positive control), but did not spread $(<5 \%$ at 28 days) in normal fibroblasts (negative control), as expected, so we used these cells for controls (Fig. 2b). AMLV-GFP spread more effeciently than GALV-GFP in osteosarcoma cells (HOS, MNNG-HOS, MG-63, Saos-2, and U2OS), although GALV-GFP also spread efficiently in MNNG-HOS cells (Fig. 2b). These results were consistent with those of RRV receptor expression in these cells. Thus, AMLV-RRV predominates over GALV-RRV in human osteosarcoma cells.

\section{Prodrug activator gene-mediated cell killing effect of RRVs in human osteosarcoma cells}

To investigate the efficacy of RRV-mediated prodrug activator gene therapy in human osteosarcoma cells, we used AMLV-CD and GALV-CD which express the CD prodrug activator gene (Fig. 3a). The human osteosarcoma cells MNNG-HOS, HOS, and MG-63 were infected with MALV-CD or GALV-CD at an MOI of 0.01 on Day 0 , and exposed to various concentrations of the 5FC prodrug from Day 15 for 3 days. On Day 18, cell viability was examined by the AlamarBlue assay. 
a

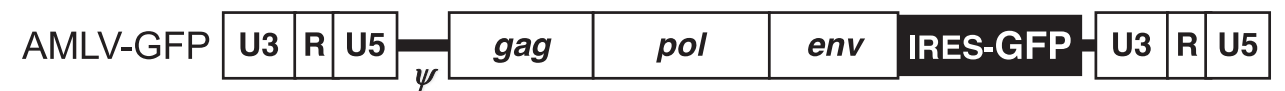

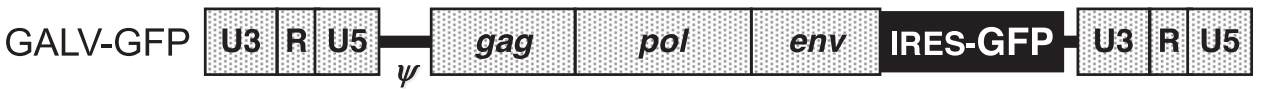

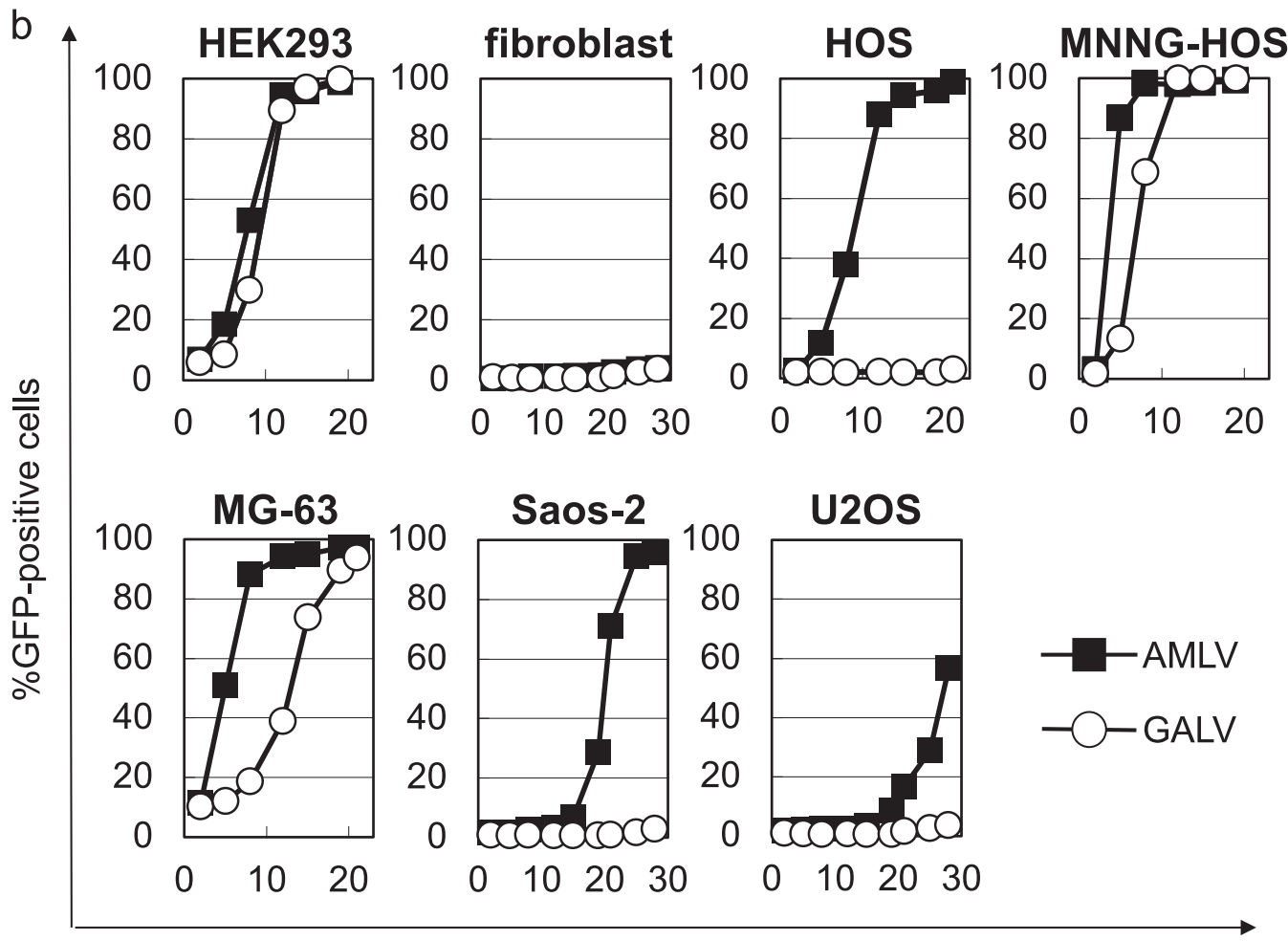

Days after infection

Fig. 2 Structure and replication kinetics of amphotropic murine leukemia virus (AMLV) vs. gibbon ape leukemia virus (GALV) vectors in. a Schematic structure of AMLV-GFP and GALV-GFP vector. These vectors contain a full-length replication-competent AMLV or GALV provirus, in which an IRES-GFP cassette has been inserted between the env gene and 3'-untranslated region. $\psi$ packaging signal, gag-pol AMLV or GALV structural genes, IRES internal ribosome entry site, GFP green fluorescent protein. b Replication kinetics of

In MNNG-HOS cells (high PiT-1 and high PiT-2 expression), decreased cell viability was observed in both AMLVCD-transduced and GALV-CD-transduced cultures in a 5FC dose-dependent manner (Fig. 3b). However, in HOS and MG-63 cells (low PiT-1 and high PiT-2 expression), significantly decreased cell viability was observed in AMLV-CD-transduced compared with GALV-CDtransduced cultures.

These results were consistent with the in vitro RRVGFP replication kinetics (Fig. 2b), and indicate that the AMLV vector could achieve selective spread and cytotoxicity in osteosarcoma cells, as well as enhanced transduction efficiency by using a different physical binding mechanism.
AMLV vs. GALV vectors in human osteosarcoma cells. Human nonmalignant cells (HEK293 cells and fibroblasts) and osteosarcoma cells (HOS, MNNG-HOS, MG-63, Saos-2, and U2OS) were inoculated with AMLV-GFP or GALV-GFP vectors at an MOI of 0.01. On the days of passage, cells were analyzed for GFP expression by flow cytometry. Data are representative of more than three independent experiments, all yielding similar results

\section{AMLV-mediated CD/5FC prodrug activator gene therapy shows potent in vivo antitumor effects in a subcutaneous human osteosarcoma xenograft model}

To examine the antitumoral therapeutic efficacy of RRVmediated $\mathrm{CD} / 5 \mathrm{FC}$ prodrug activator gene therapy, nude mice bearing established MG-63 tumors were treated with a single intratumoral injection $\left(1 \times 10^{4} \mathrm{TU}\right.$ total dose $)$ of either AMLV-CD or GALV-CD, or PBS vehicle control on Day 0 , followed by intraperitoneal administration of $5 \mathrm{FC}$. As shown in Fig. 4, subcutaneous tumors treated with GALV-CD showed no obvious inhibition of growth after 5FC administration compared with the PBS control group. 
Fig. 3 Prodrug activator genemediated cell killing effect after AMLV vs. GALV infection in vitro. a Schematic structure of AMLV-CD and GALV-CD vector. These vectors were created by replacement of the IRES-GFP cassette of AMLVGFP and GALV-GFP with IRES-CD, respectively. CD yeast cytosine deaminase prodrug activator gene. b Cell viability of RRV-infected osteosarcoma cells. MNNGHOS, HOS, and MG-63 were infected with GALV-CD or AMLV-CD at an MOI of 0.01 on Day 0 , and exposed to various concentrations of the 5 FC prodrug from Day 15 for 3 days. On Day 18, cell viability was examined by the AlamarBlue assay. Data shown are averages \pm SD from experiments performed in triplicate

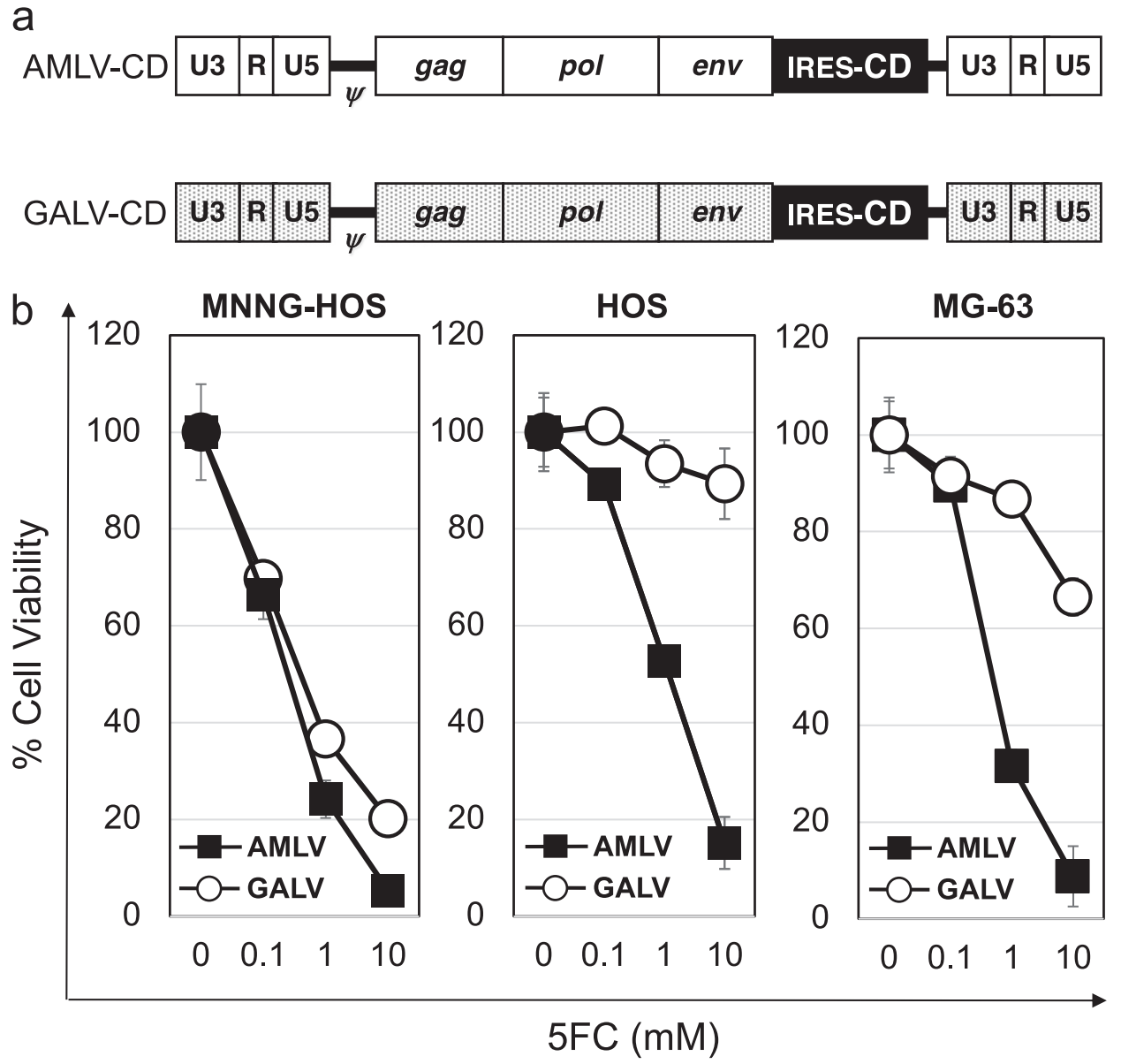

In contrast, the growth of AMLV-CD-transduced tumors was significantly inhibited by 5FC treatment $(P<0.01$ after Day 21). Thus, AMLV-mediated prodrug activator gene therapy achieved effective in vivo growth inhibition in this subcutaneous MG-63 tumor model of human osteosarcoma.

\section{Discussion}

To achieve successful prodrug activator gene therapy for cancer, efficient transduction throughout the entire tumor is required. Replicative spread of RRVs in solid tumors are potentially affected by many factors, including cell proliferation rate, antiviral innate immunity, acquired immunity, and cellular receptor expression levels. Among the factors, we previously reported that the replicative spread of RRVs in solid tumors is affected by cellular receptor expression levels in human malignant mesothelioma cells [22]. In the previous study, we detected high PiT-1 expression compared with PiT-2, suggesting that GALV predominates over AMLV in these cells, especially in ACCMESO-1 cells [22]. In this study, however, we observed low PiT-1 expression compared with PiT-2, indicating that AMLV predominates over GALV in human osteosarcoma cells. These results demonstrate that some cancer cell lines express PiT-1 and PiT-2 differentially, although the mechanisms and physiological effects of differential expression of phosphate transporters remain to be elucidated. In cells with low receptor expression, RRVs that require this receptor for viral entry showed limited replicative spread, and the efficiency of cell death after 5FC administration was correlated with RRV replication kinetics. Together with the evaluation for other factors in biopsy specimens, the use of multiple RRVs may be practically beneficial in RRV-mediated prodrug activator gene therapy against a variety of solid tumors. Moreover, personalized virotherapy based on cellular receptor expression could enhance efficacy when targeting human solid tumors.

Although most osteosarcomas have a good response to chemotherapy, $\sim 30-40 \%$ of the patients eventually develop metastases. Moreover, $>80 \%$ of relapses involve the lungs [1], and patients with pulmonary metastases at the time of diagnosis have only a $20 \%$ survival rate $[2,3,5]$. RRV has been shown to effectively transduce multiple disseminated tumors [19, 29] and achieve strong therapeutic effects in mouse models, probably due to a potent bystander effect that we previously reported using the same vector as we used in this study $[16,17]$. Hiraoka et al. reported variable 


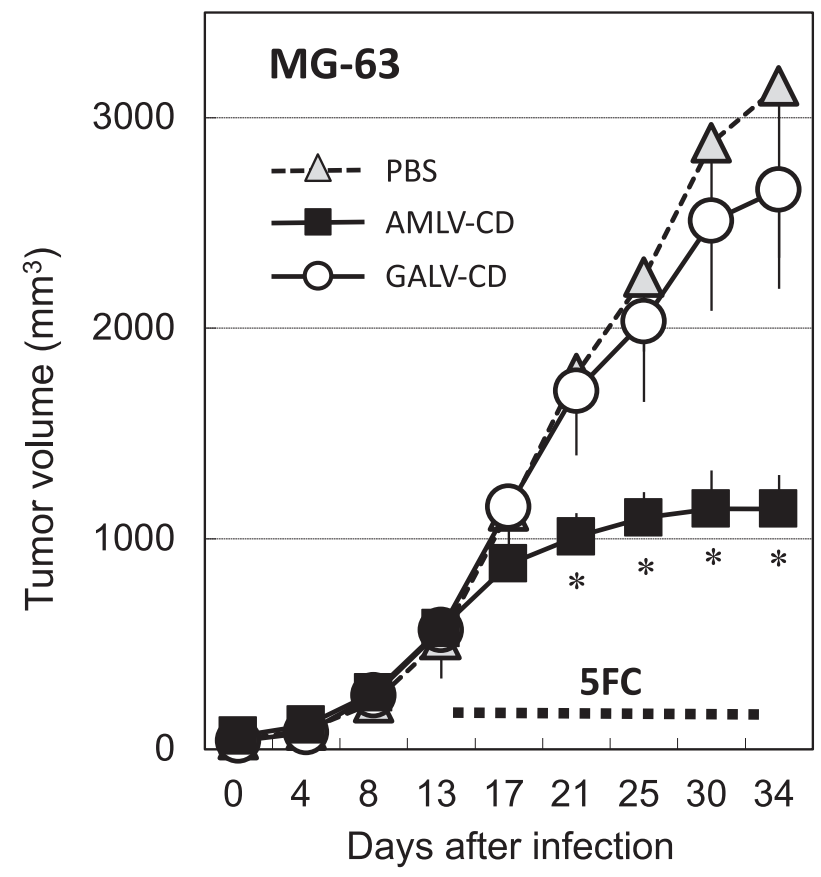

Fig. 4 In vivo antitumor effect of AMLV vs. GALV-mediated prodrug activator gene therapy in a subcutaneous xenograft model of human osteosarcoma cells. MG-63 tumors were grown subcutaneously in nude mice to $5-6 \mathrm{~mm}$ in diameter, then injected intratumorally with $1 \times 10^{4} \mathrm{TU}(50 \mu \mathrm{L})$ of either AMLV-CD or GALV-CD, or PBS vehicle control on Day 0 , followed by intraperitoneal administration of 5FC $(500 \mathrm{mg} / \mathrm{kg} /$ day $)$ three times weekly from Day 14 to Day $34(n=10$ per group). Tumor volumes were measured twice weekly, and data shown are averages-(PBS and GALV-CD) or + (AMLV-CD) SD from experiments. $* P<0.01$ (AMLV-CD vs. GALV-CD)

transduction levels among different tumor nodules after the intrasplenic injection of RRV vector in hepatic metastasis models of murine CT26 colorectal cancer [29]. Kawasaki et al. documented the efficient transduction of human malignant mesothelioma cells by RRV vector and therapeutic efficacy of $\mathrm{RRV}$-mediated $\mathrm{CD} / 5 \mathrm{FC}$ prodrug activator gene therapy in peritoneally disseminated mesothelioma models using human MSTO-211H cells pretransduced with AMLV-CD [19].

In multiple (disseminated or metastatic) tumors, the development of alternative methods for efficient in vivo systemic delivery of RRV to tumor sites is desirable, because retrovirus-based vectors are produced at only low titers in vitro, are hugely diluted in the systemic circulation, and are easily inactivated by complement in the blood. To this end, we developed a novel chimeric vector system (adenovirus-RRV hybrid vectors), in which high-titer adenoviruses were used to deliver RRV vectors. The hybrid vector system exhibited a significantly higher initial transduction and higher levels of second-stage RRV production in situ, leading to accelerated RRV vector spread and achieving enhanced therapeutic efficacy of prodrug activator gene therapy for cancer [30]. This hybrid vector may also have additional advantages for tumor targeting via fiber modification of the first-stage adenovirus [31, 32] and transcriptional regulation of second-stage RRV vectors [33]. This system therefore has great therapeutic potential to improve the clinical outcome in patients with aggressive osteosarcomas.

In conclusion, our results show that RRV vectors can efficiently replicate and achieve significant levels of tumor transduction in human osteosarcoma cells. This is the first study to show efficient transduction of human osteosarcoma cells by RRV vectors, as well as the therapeutic efficacy of AMLV vector-mediated $\mathrm{CD} / 5 \mathrm{FC}$ prodrug activator gene therapy in an MG-63 osteosarcoma model not permissive to GALV. As such, this system could be a new treatment paradigm for human osteosarcoma.

Acknowledgements We thank Atsuko Tamamoto and members of the Joint-Use Research Facilities of the Hyogo College of Medicine for their technical assistance, and Sarah Williams from Edanz Group for editing a draft of this manuscript.

Funding This work was supported by a Grant-in-Aid for Scientific Research from the Ministry of Education, Culture, Sports, Science and Technology (MEXT) of Japan (16K10484; S.K., 18H00470; M.T. K.); and the MEXT-Supported Program for the Strategic Research Foundation at Private Universities (S.K.).

\section{Compliance with ethical standards}

Conflict of interest N.K. is a paid consultant to Tocagen Inc. This does not alter the authors' adherence to all CGT policies on sharing data and materials. The remaining authors declare that they have no conflict of interest.

Open Access This article is licensed under a Creative Commons Attribution 4.0 International License, which permits use, sharing, adaptation, distribution and reproduction in any medium or format, as long as you give appropriate credit to the original author(s) and the source, provide a link to the Creative Commons license, and indicate if changes were made. The images or other third party material in this article are included in the article's Creative Commons license, unless indicated otherwise in a credit line to the material. If material is not included in the article's Creative Commons license and your intended use is not permitted by statutory regulation or exceeds the permitted use, you will need to obtain permission directly from the copyright holder. To view a copy of this license, visit http://creativecommons. org/licenses/by/4.0/.

\section{References}

1. Aljubran AH, Griffin A, Pintilie M, Blackstein M. Osteosarcoma in adolescents and adults: survival analysis with and without lung metastases. Ann Oncol. 2009;20:1136-41.

2. Harrison DJ, Geller DS, Gill JD, Lewis VO, Gorlick R. Current and future therapeutic approaches for osteosarcoma. Expert Rev Anticancer Ther. 2018;18:39-50.

3. Reed DR, Hayashi M, Wagner L, Binitie O, Steppan DA, Brohl AS, et al. Treatment pathway of bone sarcoma in children, adolescents, and young adults. Cancer. 2017;123:2206-18. 
4. Rickel K, Fang F, Tao J. Molecular genetics of osteosarcoma. Bone. 2017;102:69-79.

5. Saraf AJ, Fenger JM, Roberts RD. Osteosarcoma: accelerating progress makes for a hopeful future. Front Oncol. 2018;8:4.

6. Alemany R, Balague C, Curiel DT. Replicative adenoviruses for cancer therapy. Nat Biotechnol. 2000;18:723-7.

7. Liu TC, Kirn D. Gene therapy progress and prospects cancer: oncolytic viruses. Gene Ther. 2008;15:877-84.

8. van der Most RG, Robinson BW, Nelson DJ. Gene therapy for malignant mesothelioma: beyond the infant years. Cancer Gene Ther. 2006;13:897-904.

9. Takagi-Kimura M, Yamano T, Tagawa M, Kubo S. Oncolytic virotherapy for osteosarcoma using midkine promoter-regulated adenoviruses. Cancer Gene Ther. 2014;21:126-32.

10. Yamakawa Y, Tazawa H, Hasei J, Osaki S, Omori T, Sugiu K, et al. Role of zoledronic acid in oncolytic virotherapy: promotion of antitumor effect and prevention of bone destruction. Cancer Sci. 2017;108:1870-80.

11. Adachi Y, Reynolds PN, Yamamoto M, Wang M, Takayama K, Matsubara S, et al. A midkine promoter-based conditionally replicative adenovirus for treatment of pediatric solid tumors and bone marrow tumor purging. Cancer Res. 2001;61:7882-8.

12. Graat HC, van Beusechem VW, Schagen FH, Witlox MA, Kleinerman ES, Helder MN, et al. Intravenous administration of the conditionally replicative adenovirus Ad5-Delta24RGD induces regression of osteosarcoma lung metastases. Mol Cancer. 2008;7:9.

13. Martinez-Velez N, Xipell E, Vera B, Acanda de la Rocha A, Zalacain M, Marrodan L, et al. The oncolytic adenovirus VCN-01 as therapeutic approach against pediatric osteosarcoma. Clin Cancer Res. 2016;22:2217-25.

14. Ketola A, Hinkkanen A, Yongabi F, Furu P, Maatta AM, Liimatainen T, et al. Oncolytic Semliki forest virus vector as a novel candidate against unresectable osteosarcoma. Cancer Res. 2008; 68:8342-50.

15. Le Boeuf F, Selman M, Son HH, Bergeron A, Chen A, Tsang J, et al. Oncolytic maraba virus MG1 as a treatment for sarcoma. Int J Cancer. 2017;141:1257-64.

16. Hiraoka K, Kimura T, Logg CR, Tai CK, Haga K, Lawson GW, et al. Therapeutic efficacy of replication-competent retrovirus vector-mediated suicide gene therapy in a multifocal colorectal cancer metastasis model. Cancer Res. 2007;67:5345-53.

17. Tai CK, Wang WJ, Chen TC, Kasahara N, Single-shot, multicycle suicide gene therapy by replication-competent retrovirus vectors achieves long-term survival benefit in experimental glioma. Mol Ther. 2005;12:842-51.

18. Wang WJ, Tai CK, Kasahara N, Chen TC. Highly efficient and tumor-restricted gene transfer to malignant gliomas by replicationcompetent retroviral vectors. Hum Gene Ther. 2003;14:117-27.

19. Kawasaki Y, Tamamoto A, Takagi-Kimura M, Maeyama Y, Yamaoka N, Terada N, et al. Replication-competent retrovirus vector-mediated prodrug activator gene therapy in experimental models of human malignant mesothelioma. Cancer Gene Ther. 2011;18:571-8.
20. Ostertag D, Amundson KK, Lopez Espinoza F, Martin B, Buckley T, Galvao da Silva AP, et al. Brain tumor eradication and prolonged survival from intratumoral conversion of 5-fluorocytosine to 5-fluorouracil using a nonlytic retroviral replicating vector. J Neuro Oncol. 2012;14:145-59.

21. Perez OD, Logg CR, Hiraoka K, Diago O, Burnett R, Inagaki A, et al. Design and selection of Toca 511 for clinical use: modified retroviral replicating vector with improved stability and gene expression. Mol Ther. 2012;20:1689-98.

22. Kubo S, Takagi-Kimura M, Logg CR, Kasahara N. Highly efficient tumor transduction and antitumor efficacy in experimental human malignant mesothelioma using replicating gibbon ape leukemia virus. Cancer Gene Ther. 2013;20: 671-7.

23. Cloughesy TF, Landolfi J, Hogan DJ, Bloomfield S, Carter B, Chen CC, et al. Phase 1 trial of vocimagene amiretrorepvec and 5fluorocytosine for recurrent high-grade glioma. Sci Transl Med. 2016;8:341ra75.

24. Logg CR, Baranick BT, Lemp NA, Kasahara N. Adaptive evolution of a tagged chimeric gammaretrovirus: identification of novel cis-acting elements that modulate splicing. J Mol Biol. 2007;369:1214-29.

25. Liu M, Eiden MV. The receptors for gibbon ape leukemia virus and amphotropic murine leukemia virus are not downregulated in productively infected cells. Retrovirology. 2011;8:53.

26. Miller AD, Chen F. Retrovirus packaging cells based on 10A1 murine leukemia virus for production of vectors that use multiple receptors for cell entry. J Virol. 1996;70:5564-71.

27. Overbaugh J, Miller AD, Eiden MV. Receptors and entry cofactors for retroviruses include single and multiple transmembrane-spanning proteins as well as newly described glycophosphatidylinositol-anchored and secreted proteins. Microbiol Mol Biol Rev. 2001;65:371-89.

28. Graham FL, Smiley J, Russell WC, Nairn R. Characteristics of a human cell line transformed by DNA from human adenovirus type 5. J Gen Virol. 1977;36:59-74.

29. Hiraoka K, Kimura T, Logg CR, Kasahara N. Tumor-selective gene expression in a hepatic metastasis model after locoregional delivery of a replication-competent retrovirus vector. Clin Cancer Res. 2006;12:7108-16.

30. Kubo S, Haga K, Tamamoto A, Palmer DJ, Ng P, Okamura H, et al. Adenovirus-retrovirus hybrid vectors achieve highly enhanced tumor transduction and antitumor efficacy in vivo. Mol Ther. 2011;19:76-82.

31. Campos SK, Barry MA. Current advances and future challenges in Adenoviral vector biology and targeting. Curr Gene Ther. 2007;7:189-204

32. Glasgow JN, Bauerschmitz GJ, Curiel DT, Hemminki A. Transductional and transcriptional targeting of adenovirus for clinical applications. Curr Gene Ther. 2004;4:1-14.

33. $\operatorname{Logg}$ CR, Logg A, Matusik RJ, Bochner BH, Kasahara N. Tissuespecific transcriptional targeting of a replication-competent retroviral vector. J Virol. 2002;76:12783-91. 